\title{
Article
}

\section{Metalens Eyepiece for 3D Holographic Near-Eye Display}

\author{
Chang Wang ${ }^{1,+} \mathbb{\oplus}$, Zeqing Yu ${ }^{1,+}\left(\mathbb{C}\right.$, Qiangbo Zhang ${ }^{1}$, Yan Sun ${ }^{1}$, Chenning Tao ${ }^{1}$, Fei $\mathrm{Wu}^{2}$ and Zhenrong Zheng ${ }^{1, *}$ \\ 1 State Key Lab of Modern Optical Instrumentation, College of Optical Science and Engineering, \\ Zhejiang University, Hangzhou 310027, China; 11530032@zju.edu.cn (C.W.); 22060564@zju.edu.cn (Z.Y.); \\ 3170102974@zju.edu.cn (Q.Z.); 12130016@zju.edu.cn (Y.S.); 21630032@zju.edu.cn (C.T.) \\ 2 Beijing LLVision Technology Co., Ltd., Beijing 100000, China; wufei@llvision.com \\ * Correspondence: zzr@zju.edu.cn \\ + Those authors contributed equally to this work.
}

Citation: Wang, C.; Yu, Z.; Zhang, Q.; Sun, Y.; Tao, C.; Wu, F.; Zheng, Z. Metalens Eyepiece for 3D Holographic Near-Eye Display. Nanomaterials 2021, 11, 1920. https:// doi.org/10.3390/nano11081920

Academic Editor: Jong-Soo Lee

Received: 11 June 2021

Accepted: 12 July 2021

Published: 26 July 2021

Publisher's Note: MDPI stays neutral with regard to jurisdictional claims in published maps and institutional affiliations.

Copyright: (c) 2021 by the authors. Licensee MDPI, Basel, Switzerland. This article is an open access article distributed under the terms and conditions of the Creative Commons Attribution (CC BY) license (https:// creativecommons.org/licenses/by/ $4.0 /)$.

\begin{abstract}
Near-eye display (NED) systems for virtual reality (VR) and augmented reality (AR) have been rapidly developing; however, the widespread use of VR/AR devices is hindered by the bulky refractive and diffractive elements in the complicated optical system as well as the visual discomfort caused by excessive binocular parallax and accommodation-convergence conflict. To address these problems, an NED system combining a $5 \mathrm{~mm}$ diameter metalens eyepiece and a threedimensional (3D), computer-generated holography (CGH) based on Fresnel diffraction is proposed in this paper. Metalenses have been extensively studied for their extraordinary capabilities at wavefront shaping at a subwavelength scale, their ultrathin compactness, and their significant advantages over conventional lenses. Thus, the introduction of the metalens eyepiece is likely to reduce the issue of bulkiness in NED systems. Furthermore, CGH has typically been regarded as the optimum solution for 3D displays to overcome limitations of binocular systems, since it can restore the whole light field of the target 3D scene. Experiments are carried out for this design, where a $5 \mathrm{~mm}$ diameter metalens eyepiece composed of silicon nitride anisotropic nanofins is fabricated with diffraction efficiency and field of view for a $532 \mathrm{~nm}$ incidence of $15.7 \%$ and $31^{\circ}$, respectively. Furthermore, a novel partitioned Fresnel diffraction and resample method is applied to simulate the wave propagations needed to produce the hologram, with the metalens capable of transforming the reconstructed 3D image into a virtual image for the NED. Our work combining metalens and CGH may pave the way for portable optical display devices in the future.
\end{abstract}

Keywords: metalens; computer-generated hologram; three-dimensional near-eye display

\section{Introduction}

Metasurfaces are arrays of subwavelength dielectric or metallic antennas and have been widely investigated for their remarkable capabilities to manipulate the phase, amplitude, and polarization state of the incident light [1]. Due to their extraordinary performances in wavefront shaping, metasurfaces have been used to miniaturize various traditional optical elements into ultrathin devices, such as metalens [2-7], meta-hologram [8,9], vortex beam generator $[10,11]$, and wave plate $[12,13]$. Among those applications, metalenses are an important subcategory of metasurfaces owing to their potential replacement of conventional, bulky optical lenses with their high performance and compactness.

Near-eye display (NED) systems for virtual reality (VR) and augmented reality (AR) show considerable promise as the next generation of display technology; however, the realization of a high sense of immersion, the core requirement of VR/AR devices [14], is impeded by the bulky refractive and diffractive elements in their complicated optical systems, as well as the visual discomfort caused by excessive binocular parallax and accommodation-convergence conflicts [15]. To tackle those problems, thin optical eyepieces such as metalenses [14,16], diffractive optical elements (DOE) [17], and holographic optical elements (HOE) [18] have been investigated for their ability to miniaturize optical systems, 
and 3D computer-generated holography (CGH) was shown to have the ability to overcome the accommodation-convergence conflicts caused by binocular parallax displays [19]. However, research work integrating ultrathin optical metalens eyepiece with 3D-CGH display technology has not yet been implemented to solve both key limitations of the current NED systems.

In this paper, a display prototype system using a transparent metalens combining Fresnel-diffraction-based 3D-CGH for NED (MCGH-NED) is proposed and experimentally realized. The work initially achieved a miniaturization of the optical system into the ultrathin platform while simultaneously solving the accommodation-convergence conflict of the current NED systems. To this end, first, a $5 \mathrm{~mm}$ diameter metalens composed of silicon nitride $\left(\mathrm{Si}_{3} \mathrm{~N}_{4}\right)$ nanofin arrays on a quartz substrate was positioned directly in front of the human eye, operating at $532 \mathrm{~nm}$ incidences with high transmission, sufficient modulation efficiency, and a $31^{\circ}$ field of view (FOV). In addition, an enlarged 3D virtual image could be viewed after placing the previously mentioned 3D holographic reconstructed image (HRI) in front of the metalens eyepiece within its focal length. The 3D-CGH was implemented using a novel partitioned Fresnel diffraction and resample method based on layer-based methods $[20,21]$. Furthermore, the HRI was set left-circularly polarized (LCP) since the proposed metalens utilizes the Pancharatnam-Berry $(\mathrm{PB})$ phase manipulation and can converge a circularly polarized (CP) incident light into an output focusing light with opposite helicity [1] so that the output light can only be viewed by adding a right-circularly polarized (RCP) filter, making the proposed NED a VR display system. This work unlocks the potential of metalens and CGH display for future NED display technologies, and applications of this MCGH-NED prototype are envisioned in particular for advanced optical displays, computer vision, wearable devices, etc.

\section{Materials and Methods}

The basic working principles of the proposed MCGH-NED system that enable the transparent metalens to act as an eyepiece can be illustrated as follows. As shown in Figure 1, the transparent metalens is placed in front of the human eye, and the 3D-HRI, as the imaging object, is placed within the focal length, which is denoted by $f$, of the metalens eyepiece, where $L_{\mathrm{obj}}$ is the object size, $L_{\mathrm{img}}$ is the virtual image size, $d_{\mathrm{e}}$ is the eye relief distance, $d_{\mathrm{O}}$ is the object distance, and $d_{\mathrm{i}}$ is the virtual image distance. For this singlet lens imaging system, $d_{\mathrm{o}}$ and $d_{\mathrm{i}}$ can be set free as long as $d_{\mathrm{i}}$ is smaller than the focal length of the metalens. Then, the virtual image is modulated by the metalens eyepiece, floated to the desired location, and imaged onto the human eye or camera, where the displayed virtual image may be enlarged as needed. Thus, the FOV of the system is determined by the eyepiece size as well as the eye relief distance, rather than by the display size, which results in the metalens eyepiece being superior to conventional eyepieces [16], and the corresponding FOV can be expressed as $2 \tan ^{-1}\left[A_{\mathrm{m}} /\left(2 d_{\mathrm{e}}\right)\right]$, where $A_{\mathrm{m}}$ is metalens eyepiece aperture. Furthermore, the largest display object size of the 3D-HRI is decided by a FOV such as $f A_{\mathrm{m}} / d_{\mathrm{e}}$. It is clear from the above analysis that the desired HRI display size and the image position are linear to $f$, and the FOV of the device is directly related to $A_{\mathrm{m}}$, so the transparent metalens eyepiece should have a relatively short focal length and large aperture, which means a large numerical aperture (NA).

\subsection{Design and Fabrication of the $\mathrm{Si}_{3} \mathrm{~N}_{4}$ Metalens}

Conventional high NA objects composed of complicated compound lenses are essential in imaging, microscopy, spectroscopy, etc., but are bulky and expensive. Thus, singlet planar lenses with high NA in the visible range are in great demand, particularly compared with planar lenses made by diffractive components whose constituent structures are of wavelength scale and would therefore prevent the accurate phase profile that is of vital importance for high NA and efficiency [2]. Metalenses, planar lenses implemented by metasurfaces, are feasible to realize the designed phase profiles accurately as their constituent 
structures, or unit cells, are of subwavelength scale; hence, high NA can theoretically be achieved by metalenses.

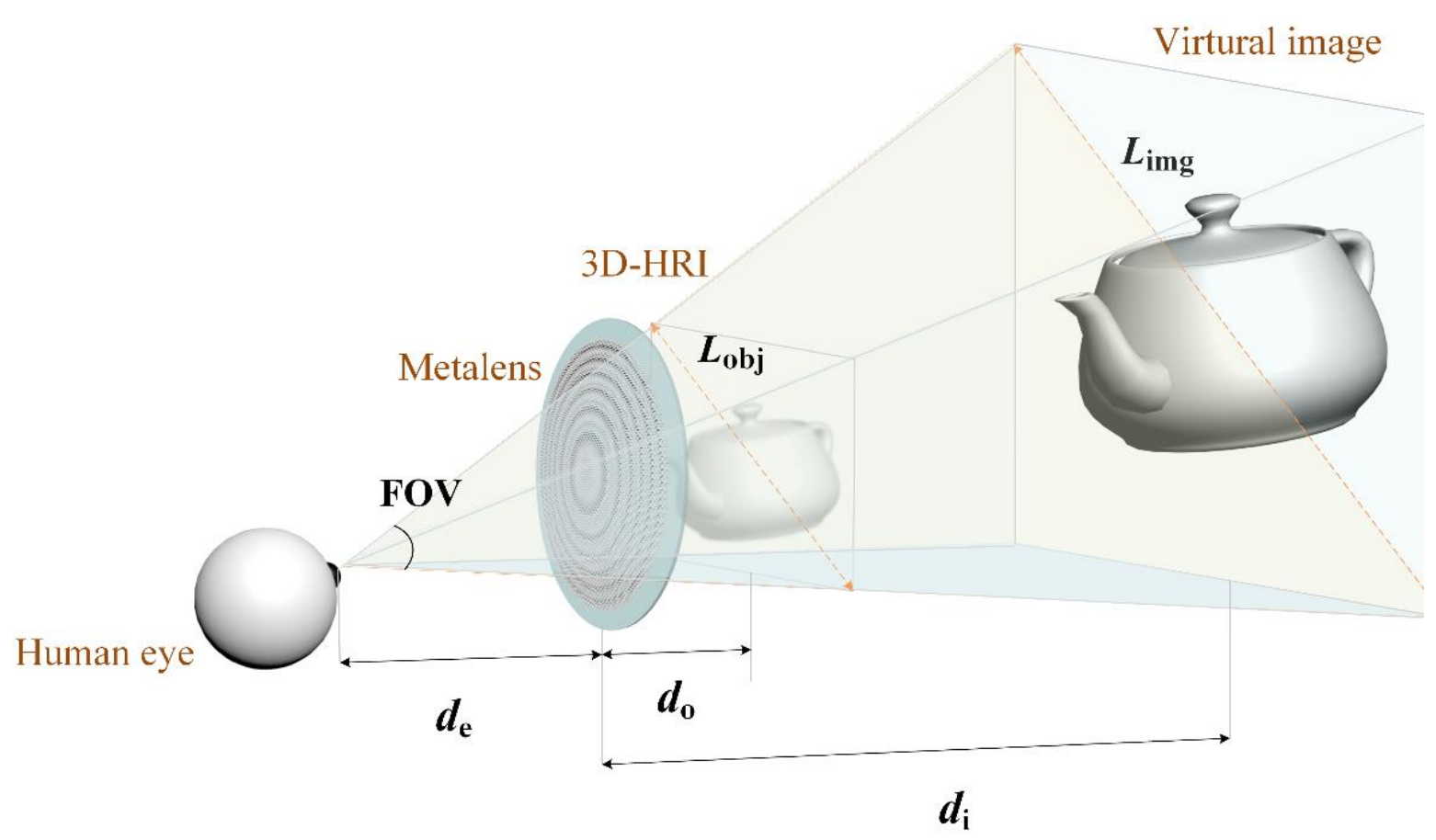

Figure 1. Schematic illustration of the proposed MCGH-NED system. An enlarged virtual image can be viewed by the human eye after placing the transparent HRI in front of the metalens eyepiece.

Optical phase discontinuity theory, derived from generalized laws of reflection and refraction [1], forms the mechanism of phase profile design for metalenses, and requires a converging phase compensation imparted on the metalens interface. The singlet metalens functions like a spherical lens, and normally its phase profile should be of a hyperboloidal form as

$$
\varphi(x, y)=2 \pi n-\frac{2 \pi}{\lambda}\left(\sqrt{\left(x-f_{x}\right)^{2}+\left(y-f_{y}\right)^{2}+f_{z}^{2}}-f\right),
$$

where $(x, y)$ represents the horizontal position along the metalens interface in the Cartesian coordinates, $\left(f_{x}, f_{y}, f_{z}\right)$ is the position of the designed focal point, $f$ is the root of quadratic sum of $f_{x}, f_{y}$ and $f_{z}$, denoting the focal length, $\lambda$ is the design wavelength, and $n$ is an arbitrary integer. In this design, the focal length and the position of the focal point are set to be $6 \mathrm{~mm}$ and $(0,0,6 \mathrm{~mm})$, respectively, and the diameter of the metalens aperture is $5 \mathrm{~mm}$, indicating an NA of 0.4. In addition, the corresponding phase profile that should be imparted along the metalens interface according to Equation (1) is shown in Figure $2 b$.

There are several phase manipulation mechanisms for realizing phase discontinuity in metalens, for instance, $\mathrm{PB}$ phase manipulation, propagation phase manipulation, plasmonic resonance tuning, etc. PB phase manipulation was selected for the metalens design because of its high accuracy of phase compensation realization [1], since it operates for $\mathrm{CP}$ incidences and realizes local phase shift by rotating nanofins of metasurface unit cells, which works similarly to half-wave plates. When a CP beam is incident on the nanofin, the transmitted light can be described by

$$
E_{t}=\frac{t_{L}+t_{S}}{2}|\sigma\rangle+\frac{t_{L}-t_{S}}{2} \exp (j 2 \sigma \theta)|-\sigma\rangle,
$$

where the spin-charge $\sigma=1$ represents LCP and $\sigma=-1$ represents RCP; $|\sigma\rangle=[1+i \sigma]^{T} / \sqrt{2}$ is the unit vector of either $\mathrm{CP} ; t_{L}$ and $t_{S}$ represent the complex transmission coefficients for linear polarized light along longer and shorter optical axes of the nanofin, respectively; 
and $\theta$ is its orientation angle along $z$ axis. For example, when such a PB metasurface is illuminated by an LCP light $(\sigma=1)$, the transmitted light consists of two components that are a co-polarized LCP light and a cross-polarized RCP light, and an additional phase shift of $2 \theta$, which is proportional only to the orientation angle, is imparted onto the RCP light. Moreover, polarization conversion efficiency (PCE) represents the proportion of the $\mathrm{CP}$ incidence that is converted to transmitted light with opposite helicity of polarization state. This efficiency is fundamentally determined by the anisotropic coefficients of $t_{L}$ and $t_{S}$, which are decided by the refractive index of the nanofin material, the structural parameters of the nanofin, and the operation wavelength.
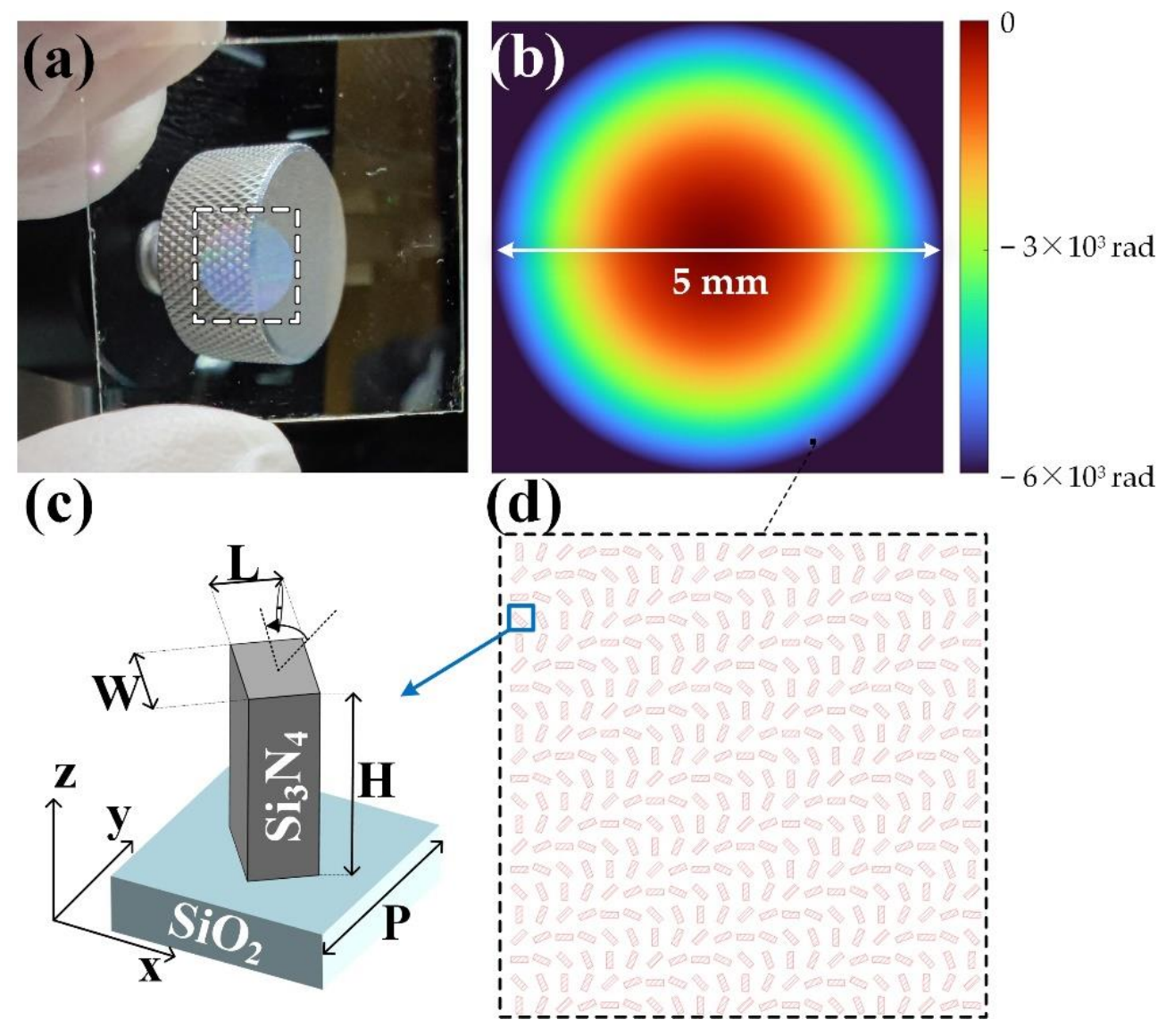

Figure 2. (a) The appearance of the fabricated transparent $\mathrm{Si}_{3} \mathrm{~N}_{4}$ metalens eyepiece. (b) The phase profile of the designed metalens. (c) $\mathrm{Si}_{3} \mathrm{~N}_{4}$ nanofin on quartz substrate as metalens unit cell. (d) Top view of local patterns of the nanofins, rotating to realize the desired PB phases in (b).

The complex refractive index of the material used for the metalens nanofins is of significance for the PCE of single nanofins and the focusing efficiency of the whole metalens. $\mathrm{Si}_{3} \mathrm{~N}_{4}$ is chosen for this design due to its exceptionally low extinction coefficient $(k)$, large refractive index $(n)$ at visible wavelengths, and CMOS-compatible fabrication [2]. Figure 2c illustrates the selected metalens unit cell, consisting of a $\mathrm{Si}_{3} \mathrm{~N}_{4}$ nanofin of high aspect-ratio and a layer of quartz substrate. The height of this nanofin is $400 \mathrm{~nm}$, and the actually measured refractive index has an $n$ of 1.99 and $k$ of about zero at a $532 \mathrm{~nm}$ wavelength after growing a $400 \mathrm{~nm}$ thick $\mathrm{Si}_{3} \mathrm{~N}_{4}$ layer for fabrication. Next, the optimization of other structural parameters for an improved PCE of the nanofin was carried out by full-wave simulations using a commercial package of Ansys Lumerical FDTD Solutions 2020 R2, with an AMD Ryzen Threadripper 3990X CPU (2.9 GHz) with 256 GB RAM utilized as the corresponding computing platform. For the metalens nanofin simulations, periodic boundary condition was applied in the $x$-direction and $y$-direction and perfect matching layer (PML) boundary condition was applied at z-direction, with the incidence being set 
as a $532 \mathrm{~nm}$ collimated LCP light. The corresponding computation time for each nanofin simulation was approximately $13-15 \mathrm{~s}$.

It is worth noting that for the design of the whole metalens, the values of the converging phase function as expressed in Equation (1) were directly applied to each nanofin by PB phase manipulation, without considering the local interactions of neighboring nanofins. For this dielectric metalens, although the induced optical fields would theoretically be highly concentrated inside the dielectric nanofins due to their waveguide-like cavity resonances [22], such local interactions are not entirely negligible and may degrade the overall behavior of the designed metalens to some extent.

\subsection{Methods of $3 D C G H$}

In computer holography, the relation between the source and the destination planes can be given by Fresnel diffraction, the approximation form of Rayleigh-Sommerfeld diffraction, which is written as

$$
U_{2}\left(x_{2}, y_{2}\right)=\mathcal{F}^{-1}\left\{\mathcal{F}\left[U_{1}\left(x_{1}, y_{1}\right)\right] H\left(f_{x}, f_{y}\right)\right\},
$$

where $U_{1}\left(x_{1}, y_{1}\right)$ and $U_{2}\left(x_{2}, y_{2}\right)$ represent the complex amplitude of the source plane and the destination plane respectively. The transfer function in Fresnel diffraction is defined as

$$
H\left(f_{x}, f_{y}\right)=\exp (j k z) \exp \left(-j \pi \lambda z\left(f_{x}{ }^{2}+f_{y}{ }^{2}\right)\right),
$$

where $z$ is the propagation distance, $f_{x}=x /(\lambda z)$ and $f_{y}=y /(\lambda z)$. The MCGH-NED utilizes a novel partitioned Fresnel diffraction method, whose hologram is spatially segmented into multiple partitions as sub-holograms generated from different images. $L_{\mathrm{sub} x}=L_{x} / M$ and $L_{\text {suby }}=L_{y} / N$ are defined as the width and the height of sub-holograms, respectively, where $L_{x}$ and $L_{y}$ denote the width and height of CGH plane, respectively. $M$ and $N$ are the number of segments along $x$ and $y$ directions. Then the denotations of $x_{m}=[m-(M-1) / 2] \cdot L_{\text {subx }}$ and $y_{n}=[n-(N-1) / 2] \cdot L_{\text {suby }}$ are derived to represent the center coordinates of each subholograms, where $m$ is an integer from 1 to $M$ and $n$ is an integer from 1 to $N$, respectively.

Figure 3, above, demonstrates an example of the partitioned Fresnel diffraction method. Assuming that $U_{2 \mathrm{sub}}\left(x_{2 \mathrm{sub}}, y_{2 \mathrm{sub}}\right)$ in Figure 3a represents the original image of the number " 1 ", $U_{1 \text { sub }}\left(x_{1 \text { sub }}, y_{1 \text { sub }}\right)$ is the sub-hologram produced from $U_{2 \text { sub }}\left(x_{2 \text { sub }}\right.$, $\left.y_{2 \mathrm{sub}}\right)$ as depicted in Figure $3 \mathrm{~b}$. When this sub-hologram is shifted to the location of $\left(x_{m}, y_{n}\right)$ as shown in Figure 3c, Equation (3) can be derived as

$$
\begin{aligned}
U_{2 \text { sub }}\left(x_{2 \text { sub }}, y_{2 \text { sub }}\right) & =\mathcal{F}^{-1}\left\{\mathcal{F}\left[U_{1 \text { sub }}\left(x_{1 \text { sub }}-x_{m}, y_{1 \text { sub }}-y_{n}\right)\right] H\left(f_{x}, f_{y}\right)\right\} \\
& =\mathcal{F}^{-1}\left\{\mathcal{F}\left[U_{1 \text { sub }}\left(x_{1 \text { sub }}, y_{1 \text { sub }}\right)\right] H\left(f_{x}, f_{y}\right) \exp \left[-j 2 \pi\left(f_{x} x_{m}+f_{y} y_{n}\right)\right]\right\} .
\end{aligned}
$$

According to the convolution theorem, Equation (5) can be rewritten as

$$
\begin{aligned}
U \prime_{2 \text { sub }}\left(x_{2 \text { sub }}, y_{2 \text { sub }}\right) & =U_{2 \text { sub }}\left(x_{2 \text { sub }}, y_{2 \text { sub }}\right) \otimes \delta\left(x_{2 \text { sub }}-x_{m}, y_{2 \text { sub }}-y_{n}\right) \\
& =U_{2 \text { sub }}\left(x_{2 \text { sub }}-x_{m}, y_{2 \text { sub }}-y_{n}\right),
\end{aligned}
$$

where $U_{2 \text { sub }}^{\prime}\left(x_{2 \mathrm{sub}}, y_{2 \mathrm{sub}}\right)$ is the reconstruction of the shifted $U_{1 \mathrm{sub}}\left(x_{1 \mathrm{sub}}, y_{1 \text { sub }}\right)$, proving that the reconstruction in Figure $3 \mathrm{~d}$ is of the same size and location as its sub-hologram in Figure 3c.

For a more complex 3D scene, multiple layers are produced by slicing the 3D scene with various contents at the same position. Harnessing no overlap between different layers in the hologram, the proposed method eliminates the crosstalk between layers that decreases the reconstruction quality in conventional layer-based methods. However, if the reconstructed image shifts with the location of the sub-hologram rather than being at the center, a broken 3D scene would appear. To correct the position of the reconstruction at the center, a resample method is used as explained in Figure 4. In this demonstration, the original image is a 3D model "teapot", which is then sliced into three layers according to the depth to form 3 sub-images. The hologram in Figure $4 \mathrm{~b}$ consists of three sub-holograms 
generated from sub-images in Figure 4a. As Figure 4c depicts, pixels at the same relative positions in 3 sub-holograms are picked to form a three-pixel unit that is set in a side-by-side way in a new hologram as shown in Figure 4d,e.
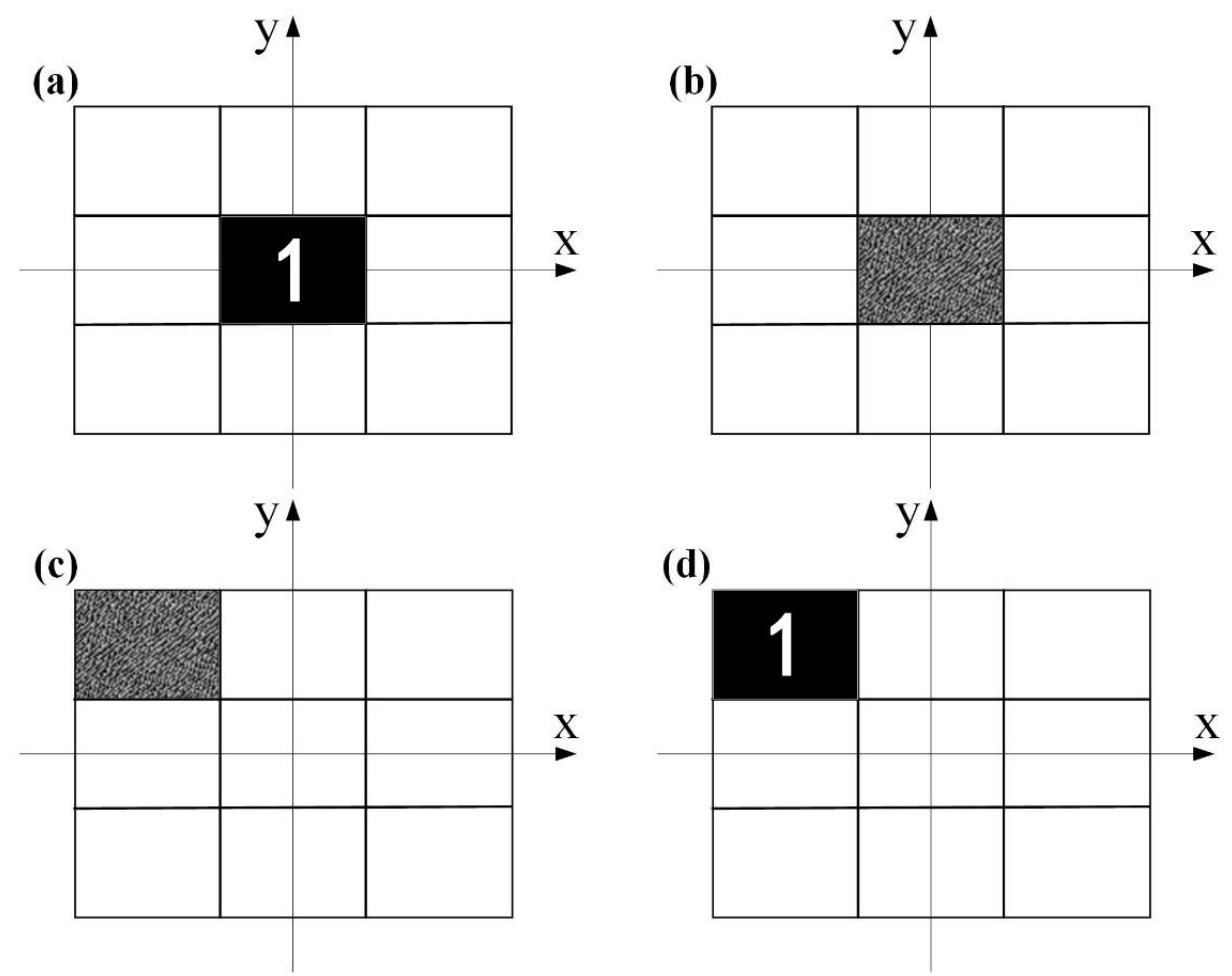

Figure 3. Example of the proposed partitioned Fresnel diffraction method. (a,b) Original sub-image and the sub-hologram. (c) Sub-hologram shifted to another location. (d) The reconstructed image after shift.

(a)

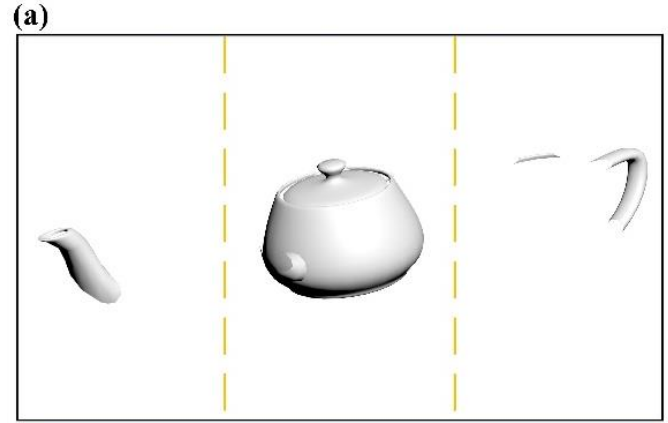

(c)

(b)
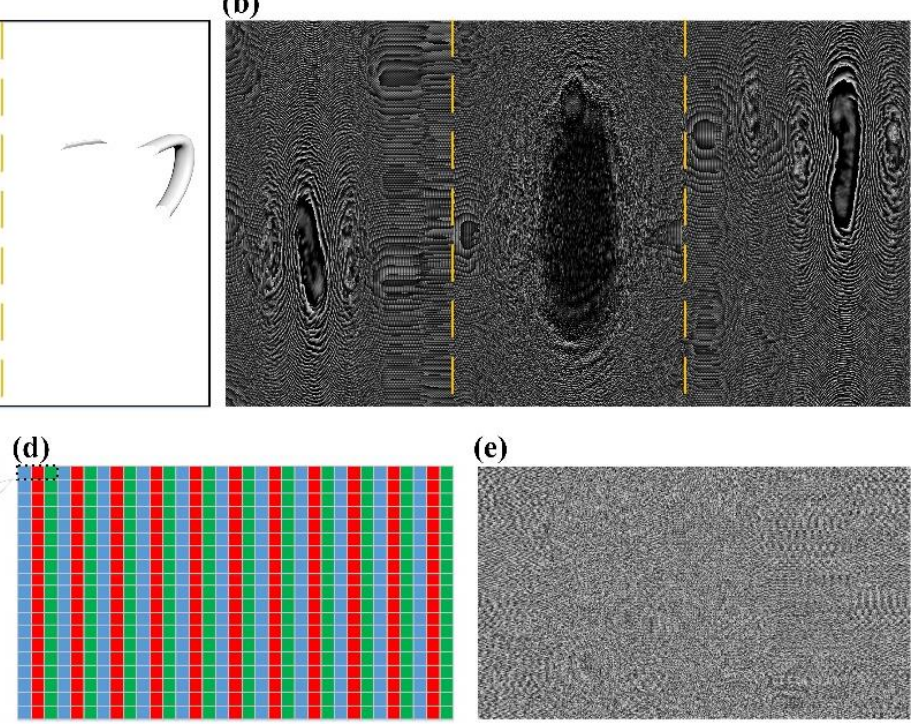

(e)
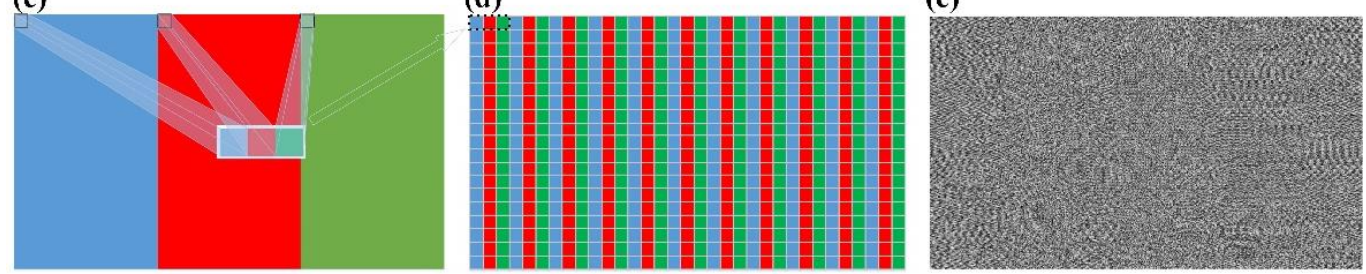

Figure 4. The proposed resample method. $(\mathbf{a}, \mathbf{b})$ The original image consisting of three sub-images sliced from a 3D model "teapot" and the corresponding hologram. (c) Three sub-holograms and a three-pixel unit composed of 3 pixels from 3 sub-holograms at the same position. (d) Three-pixel units arranged in a side-by-side fashion. (e) The hologram generated from $(b)$ through the resample method. 


\section{Results and Discussion}

As a result of the optimization for adequate PCE by FDTD simulations, each nanofin has a high aspect ratio and a rectangular cross-section, with longer length $L$, shorter length $W$, and periodic spacing $P$ of $300 \mathrm{~nm}, 105 \mathrm{~nm}$ and $400 \mathrm{~nm}$, while the simulated PCE is $24.3 \%$ under a $532 \mathrm{~nm}$ incident light. The subsequent fabrication processes are carried out as follows: the growth of a $400 \mathrm{~nm}$ thick $\mathrm{Si}_{3} \mathrm{~N}_{4}$ layer on a double-polished quartz plate is prepared by using plasma-enhanced chemical vapor deposition (PECVD), and subsequently a layer of ZEP-520A e-beam resist is spin-coated on it. This sample is then exposed through electron-beam lithography (EBL) to define the structure patterns, which are revealed after the development process of the resist. Next, a $100 \mathrm{~nm}$ thick $\mathrm{Cr}$ layer is deposited on the sample as a hard-etching mask by electron gun evaporation, and a lift-off process is followed. After the removal of the resist, the required patterns are transferred to the $\mathrm{Cr}$ hard mask, and the final sample is obtained by reactive ion etching (RIE) and the removal of the patterned $\mathrm{Cr}$ hard mask. Figure 5, below, shows the characterizing results of the fabricated metalens.
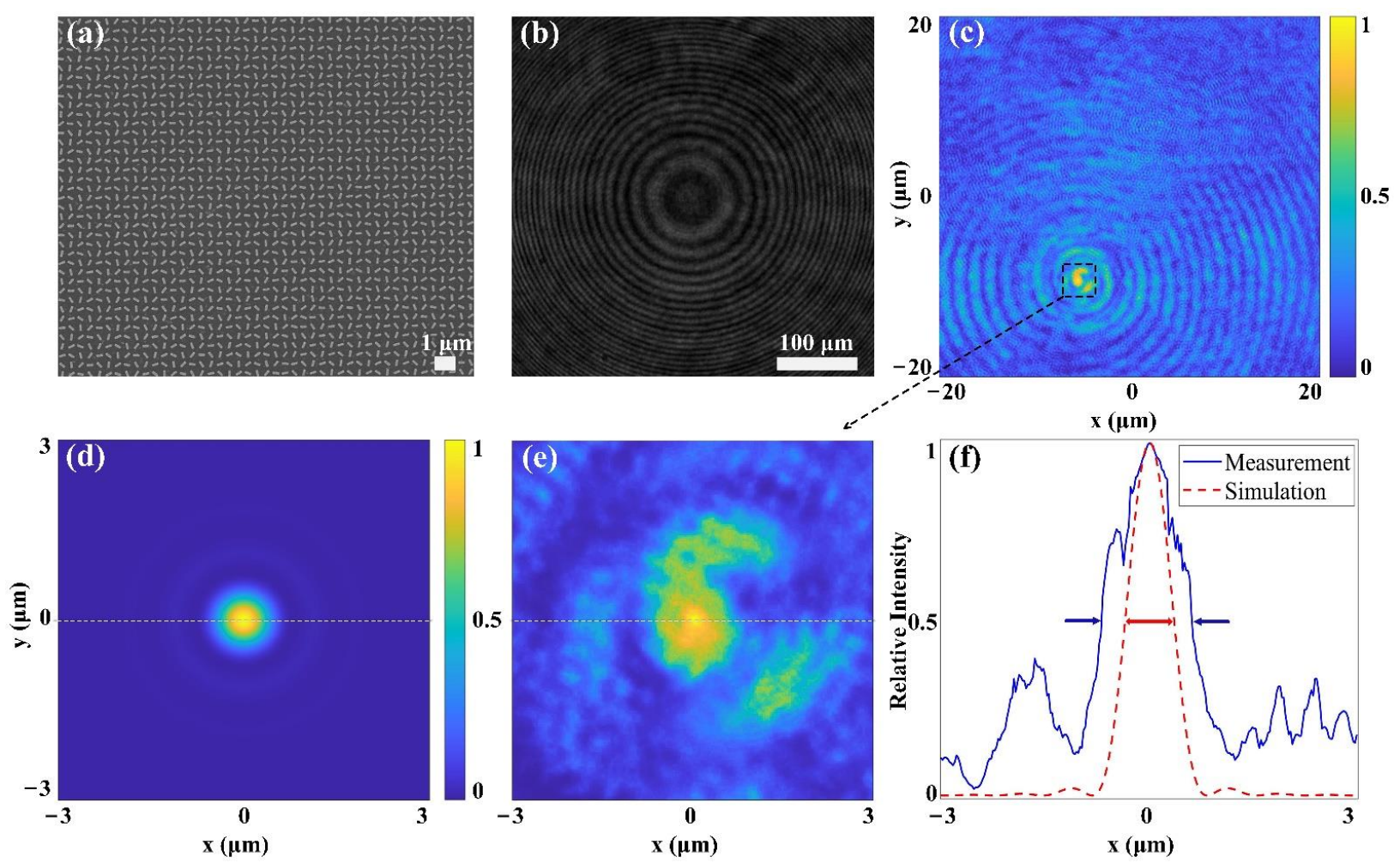

Figure 5. The characterizations of the fabricated singlet metalens. (a) A scanning electron micrograph (SEM) image of the local part from the fabricated metalens. (b) Optical image of the central part from the fabricated metalens under $532 \mathrm{~nm}$ microscopic illuminating light. (c) Measured focal spot intensity profile at $532 \mathrm{~nm}$ wavelength by a commercial object $(100 \times$ OLYMPUS, NA = 0.90). (d) Simulated focal spot intensity profile at $532 \mathrm{~nm}$ wavelength by FDTD method. (e) Magnified image of the measured focal spot intensity profile from (c). (f) Central focal spot intensity profiles along horizontal axis from (d) and (e), respectively.

Figure 5a represents the scanning electron micrograph (SEM) image of the fabricated singlet metalens, and Figure $5 \mathrm{~b}$ shows the microscopic image of the fabricated metalens under $532 \mathrm{~nm}$ laser illumination. For Figure $5 \mathrm{c}$, the corresponding full-width at halfmaximum (FWHM) measured at $532 \mathrm{~nm}$ LCP incidence is $1.49 \mu \mathrm{m}$, approximately 2.2 times the diffraction limit $(\lambda /(2 \mathrm{NA})=0.67 \mu \mathrm{m})$, and the focusing efficiency of the fabricated metalens, defined as the focusing power from a circular area with a diameter of three times FWHM over the incident power, is $15.7 \%$ when measured under a $532 \mathrm{~nm}$ LCP incidence. 
In addition, in order to compare the focusing characteristics between the simulation and the measurement, the simulated electric field intensity distribution of the focal plane for a minimized metalens, which has a relatively small aperture diameter but the same NA of 0.4 as the fabricated metalens, is shown in Figure 5d. In this case, the simulated minimized metalens has an aperture diameter of $60 \mu \mathrm{m}$ with a focal length of $64 \mu \mathrm{m}$, and PML boundary condition is applied at $\mathrm{x}$-direction, $\mathrm{y}$-direction, and $\mathrm{z}$-direction, with the corresponding computation time for such minimized metalens being about $5 \mathrm{~h}$ and $18 \mathrm{~min}$. There are two reasons for simulating such a minimized metalens: first, the designed metalens of a $5 \mathrm{~mm}$ diameter aperture is composed of over $1.22 \times 10^{8} \mathrm{Si}_{3} \mathrm{~N}_{4}$ nanofins, demanding excessive computation resources for FDTD simulations in current computing stations; second, when two metalenses of different apertures but the same NA of 0.4 are illuminated under unchanged incident light, their focal spots and the corresponding diffraction limits will theoretically be identical, indicating the same focusing characteristics.

The simulated focal spot intensity profile of such minimized metalens is shown in Figure $5 \mathrm{~d}$, and a comparison of the central focal spot intensity profiles along the horizontal axis from Figure $5 d$,e, which is a magnified picture of Figure $5 c$, is illustrated in Figure $5 f$. The simulated FWHM for such a minimized metalens with the same NA is about $0.83 \mu \mathrm{m}$, far smaller than that of the measured FWHM of the fabricated metalens, which is $1.49 \mu \mathrm{m}$, as mentioned above. Factors such as fabrication errors and local interactions among neighboring metalens nanofins would all account for the degrading of the behavior of the fabricated metalens.

Furthermore, in order to characterize the performance of the proposed metalens in the 3 D holographic display, optical imaging experiments were carried out and the experimental setup for the MCGH-NED system is demonstrated in Figure 6. First, a collimated $532 \mathrm{~nm}$ laser beam is deflected by a polarization beam splitter (PBS) and hits a phase-only spatial light modulator (SLM), which is a liquid crystal on silicon (LCoS, Holo-eye LETO), as the image engine. This LCoS has a resolution of $1920 \times 1080$ with the pixel pitch being $6.4 \mu \mathrm{m}$. After the hologram-loaded LCoS is illuminated by the laser source the reconstruction is generated and the 3D HRI transmits through an optical 4-F system, composed of two lenses of $200 \mathrm{~mm}$ and $100 \mathrm{~mm}$ focal lengths with a pinhole between them. The HRI is thus shrunk by a factor of 0.5 in order to fit the maximum display size of the metalens eyepiece, and the measured eye relief is $9 \mathrm{~mm}$, indicating a FOV of $31^{\circ}$.

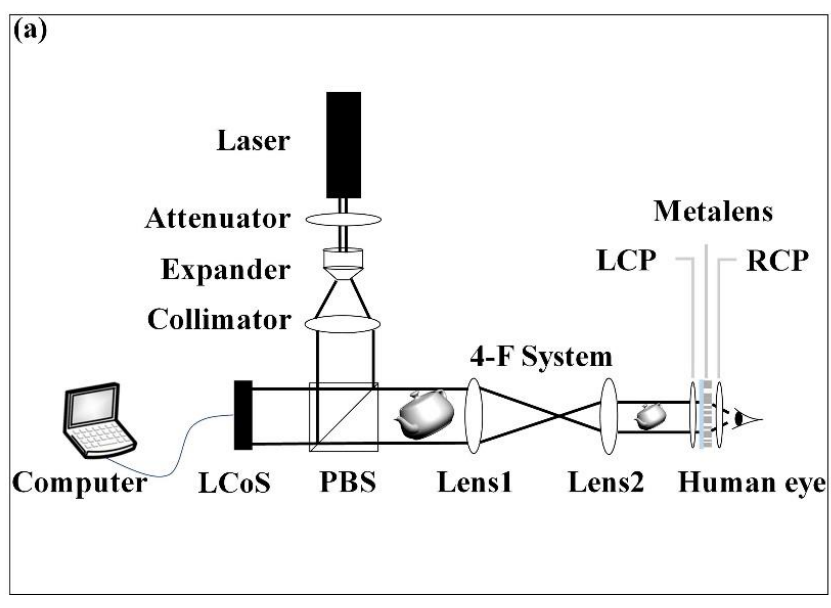

Figure 6. (a) Schematic of the MCGH-NED experimental setup.

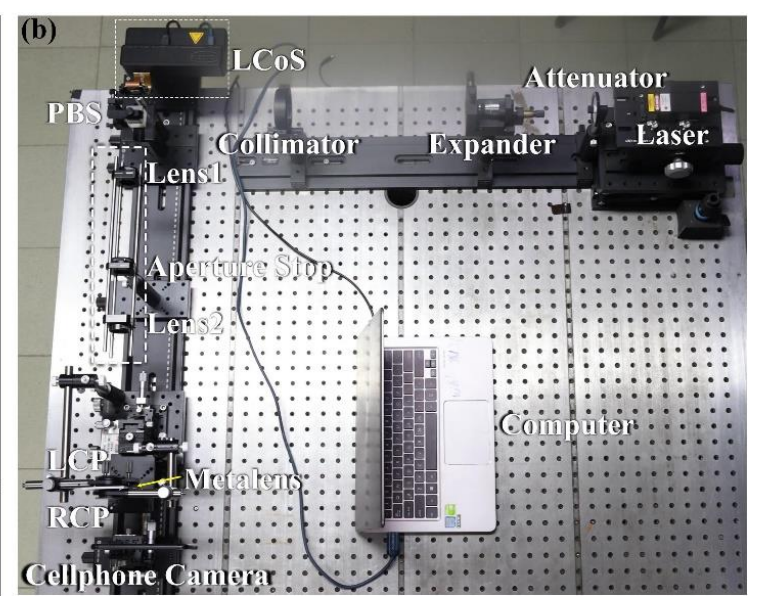

(b) Photograph of the MCGH-NED experimental setup.

As for the problem of zero-order light, the digital blazed grating is introduced in the hologram. It can be expressed as $\varphi_{\mathrm{bg}}(x, y)=(2 \pi / T) \cdot \bmod (c x+d y, T)$, where $T$ is the period of digital blazed grating, and $c$ and $d$ are two constants related to the shift distance in $x$ and $y$ directions, respectively. For this system, $c$ as well as $d$ are 0 and 1 , and $T$ is 1.5 times the sampling interval of the hologram. After the modulation of digital blazed grating, the HRI can be separated from zero-order light, which can be eliminated by the 
pinhole at the confocal position of the 4-F system for suppressing noise. Ultimately, the transferred 3D HRI is positioned within the focal length of the metalens, and an LCP polarizer is placed between it and the metalens eyepiece, which can focus LCP incidence and output focused RCP light. Having been modulated by the metalens, the output light passes through an RCP polarizer for filtering the desired virtual image and realizing the VR display. The floated virtual image is then viewed by the human eye and captured by an iPhone 6 cellphone camera.

Figure 7 above shows the experimental results of a 3D holographic near-eye display with the designed metalens as an eyepiece. Figure $7 \mathrm{a}$ is the original layered model of ZJU. Three sub-holograms are calculated from Z, J, and U, respectively, with different propagation distances, and the propagation distance interval is set at $100 \mathrm{~mm}$. The images are reconstructed at different distances, and as a result, the characters $Z, J$, and $U$ are separately focused at corresponding positions, as shown in Figure $7 \mathrm{~b}-\mathrm{d}$. It can be observed that when one of the characters is focused by the metalens eyepiece, the other two characters are blurred.
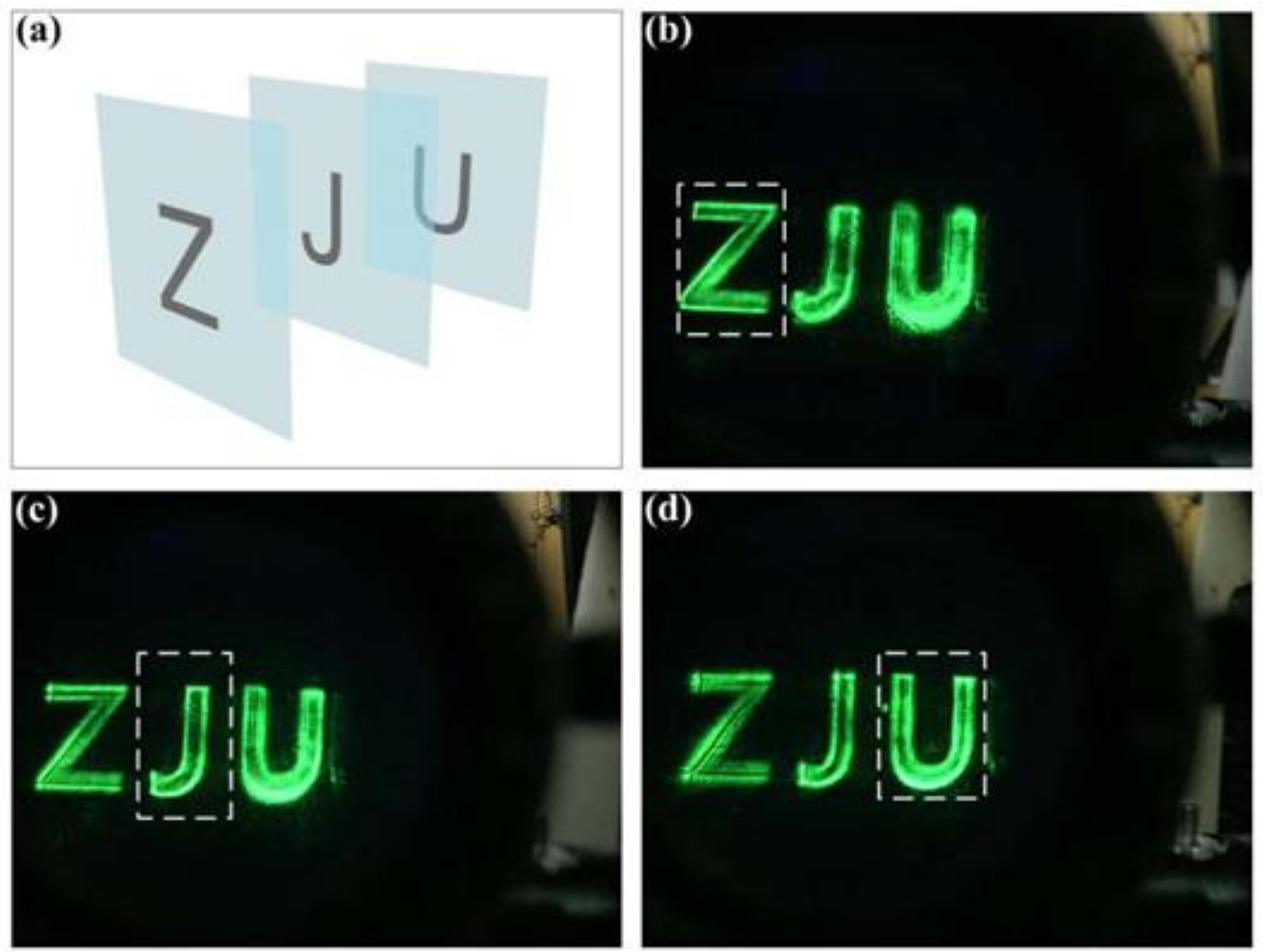

Figure 7. 3D near-eye display of ZJU characters. (a) The layered model ZJU. (b-d) The reconstructed images focused at Z, J, and $U$, respectively.

In Figure 8 below, a complex 3D model, "teapot", is displayed and observed experimentally. As Figure 8a illustrates, the model is sliced into three layers and the spacing between neighboring layers is $100 \mathrm{~mm}$. Then the holograms are produced from these layers according to the resample method mentioned above, and Figure $8 \mathrm{~b}-\mathrm{d}$ are the captured virtual images of the HRI imaged by the metalens eyepiece. It can be seen that the spout of the teapot is focused in Figure $8 \mathrm{~b}$, while the lid and the handle are blurred. In contrast, the lid and the handle in Figure 8c,d are focused respectively, while the remaining two parts are blurred when viewing through the metalens eyepiece. 


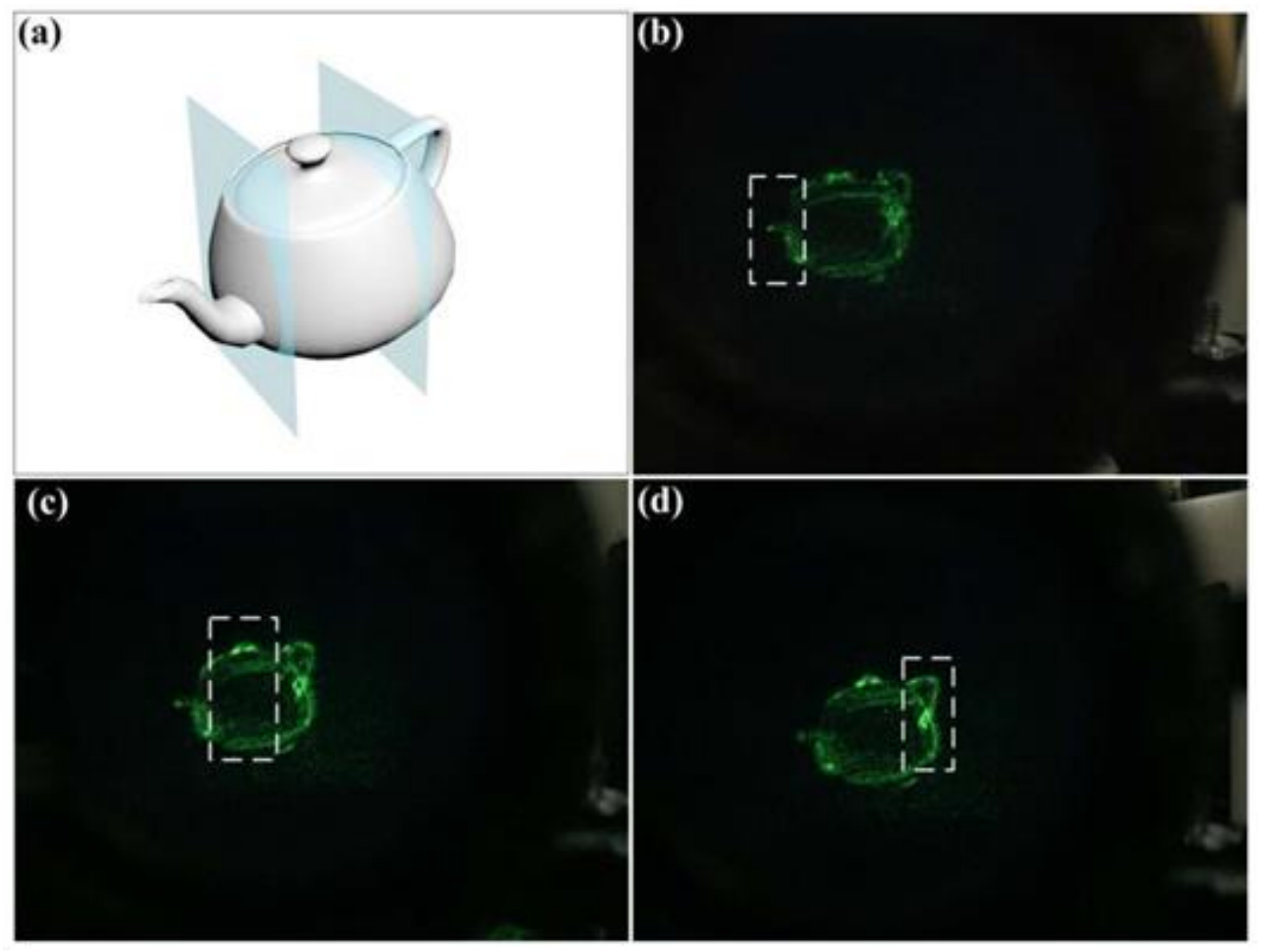

Figure 8. Experimental results of 3D near-eye display of "teapot". (a) The sliced 3D model "teapot". (b-d) The reconstruction focused on the spout, lid and handle, respectively.

\section{Conclusions}

In this paper, a holographic 3D-NED system using a transparent metalens as an eyepiece, named MCGH-NED, was proposed. Featuring a subwavelength-scale wavefront manipulation, ultrathin size, and high-performance features, a metalens eyepiece with $15.7 \%$ focusing efficiency, a diameter of $5 \mathrm{~mm}$, and an NA of 0.4 was designed and fabricated to overcome the issues of bulkiness and limited FOV in the NED system. Furthermore, a CGH technique developed by a novel partitioned Fresnel diffraction and resample method was utilized to demonstrate the display characteristics of the metalens-based 3D-NED system, confirming the advance of 3D-CGH display over traditional binocular NED displays. Experimental results demonstrated a $31^{\circ} \mathrm{FOV}$ of the MCGH-NED system and clear 3D image display performance of the metalens as an eyepiece for observing the 3D-CGH virtual images. Furthermore, the $\mathrm{CP}$ light-converting property of the proposed metalens eyepiece realizes the VR display mode. This work takes the initiative in integrating metalens and 3D-CGH displays in NED systems, and may hopefully find wide application in advanced and compact optical display technologies in the near future.

Author Contributions: Conceptualization, C.W., Z.Y. and Z.Z.; methodology, C.W., Z.Y. and Z.Z.; software, C.W.; validation, C.W. and Z.Y.; formal analysis, C.W. and Z.Y.; investigation, C.W., Z.Y., Q.Z., Y.S. and C.T.; resources, C.W., Z.Y. and Z.Z.; writing-original draft preparation, C.W., Z.Y., Q.Z., Y.S., C.T., F.W. and Z.Z.; writing—-review and editing, C.W., Z.Y. and Z.Z.; supervision, Z.Z.; funding acquisition, F.W. and Z.Z. All authors have read and agreed to the published version of the manuscript. 
Funding: This research was funded by the National Natural Science Foundation of China (NSFC) under Grant Nos. 61327902 and Beijing Municipal Science and Technology Commission (BMSTC) under Grant Nos. Z201100004020012.

Institutional Review Board Statement: Not applicable.

Informed Consent Statement: Not applicable.

Data Availability Statement: No new data were created or analyzed in this study. Data sharing is not applicable to this article.

Conflicts of Interest: The authors declare no conflict of interest.

\section{References}

1. Yu, N.; Capasso, F. Flat optics with designer metasurfaces. Nat. Mater. 2014, 13, 139-150. [CrossRef] [PubMed]

2. Khorasaninejad, M.; Chen, W.T.; Devlin, R.C.; Oh, J.; Zhu, A.Y.; Capasso, F. Metalenses at visible wavelengths: Diffraction-limited focusing and subwavelength resolution imaging. Science 2016, 352, 1190-1194. [CrossRef]

3. Zuo, H.; Choi, D.Y.; Gai, X.; Ma, P.; Xu, L.; Neshev, D.N.; Zhang, B.; Luther-Davies, B. High-efficiency all-dielectric metalenses for mid-infrared imaging. Adv. Opt. Mater. 2017, 5, 1700585. [CrossRef]

4. Wang, S.; Wu, P.C.; Su, V.-C.; Lai, Y.-C.; Hung Chu, C.; Chen, J.-W.; Lu, S.-H.; Chen, J.; Xu, B.; Kuan, C.-H.; et al. Broadband achromatic optical metasurface devices. Nat. Commun. 2017, 8, 187. [CrossRef]

5. Chen, W.T.; Zhu, A.Y.; Sanjeev, V.; Khorasaninejad, M.; Shi, Z.; Lee, E.; Capasso, F. A broadband achromatic metalens for focusing and imaging in the visible. Nat. Nanotechnol. 2018, 13, 220-226. [CrossRef] [PubMed]

6. Arbabi, E.; Arbabi, A.; Kamali, S.M.; Horie, Y.; Faraji-Dana, M.; Faraon, A. Mems-tunable dielectric metasurface lens. Nat. Commun. 2018, 9, 812. [CrossRef] [PubMed]

7. Colburn, S.; Zhan, A.; Majumdar, A. Varifocal zoom imaging with large area focal length adjustable metalenses. Optica 2018, 5, 825-831. [CrossRef]

8. Li, L.; Cui, T.J.; Ji, W.; Liu, S.; Ding, J.; Wan, X.; Li, Y.B.; Jiang, M.; Qiu, C.-W.; Zhang, S. Electromagnetic reprogrammable coding-metasurface holograms. Nat. Commun. 2017, 8, 197. [CrossRef] [PubMed]

9. Zheng, G.; Mühlenbernd, H.; Kenney, M.; Li, G.; Zentgraf, T.; Zhang, S. Metasurface holograms reaching $80 \%$ efficiency. Nat. Nanotechnol. 2015, 10, 308-312. [CrossRef]

10. Shitrit, N.; Bretner, I.; Gorodetski, Y.; Kleiner, V.; Hasman, E. Optical spin Hall effects in plasmonic chains. Nano Lett. 2011, 11, 2038-2042. [CrossRef]

11. Yang, Y.; Wang, W.; Moitra, P.; Kravchenko, I.I.; Briggs, D.P.; Valentine, J. Dielectric meta-reflect array for broadband linear polarization conversion and optical vortex generation. Nano Lett. 2014, 14, 1394-1399. [CrossRef]

12. Zhao, Y.; Alù, A. Tailoring the Dispersion of Plasmonic Nanorods to Realize Broadband Optical Meta-Waveplates. Nano Lett. 2013, 13, 1086-1091. [CrossRef] [PubMed]

13. Kim, J.; Choudhury, S.; DeVault, C.; Zhao, Y.; Kildishev, A.V.; Shalaev, V.M.; Alù, A.; Boltasseva, A. Controlling the polarization state of light with plasmonic metal oxide metasurface. ACS Nano 2016, 10, 9326-9333. [CrossRef]

14. Li, Z.; Lin, P.; Huang, Y.W.; Park, J.S.; Chen, W.T.; Shi, Z.; Qiu, C.W.; Cheng, J.X.; Capasso, F. Meta-optics achieves RGB-achromatic focusing for virtual reality. Sci. Adv. 2021, 7, 4458. [CrossRef] [PubMed]

15. Hoffman, D.M.; Girshick, A.R.; Akeley, K.; Banks, M.S. Vergence-accommodation conflicts hinder visual performance and cause visual fatigue. J. Vis. 2008, 8, 33. [CrossRef]

16. Lee, G.Y.; Hong, J.Y.; Hwang, S.; Moon, S.; Kang, H.; Jeon, S.; Kim, H.; Jeong, J.H.; Lee, B. Metasurface eyepiece for augmented reality. Nat. Commun. 2018, 9, 4562. [CrossRef]

17. Levola, T. Diffractive optics for virtual reality displays. J. Soc. Inf. Disp. 2006, 14, 467-475. [CrossRef]

18. Maimone, A.; Georgiou, A.; Kollin, J.S. Holographic near-eye displays for virtual and augmented reality. ACM Trans. Graph. 2017, 36, 85. [CrossRef]

19. He, Z.; Sui, X.; Jin, G.; Cao, L. Progress in virtual reality and augmented reality based on holographic display. Appl. Opt. 2019, 58, A74-A81. [CrossRef] [PubMed]

20. Zheng, H.; Yu, Y.; Wang, T.; Dai, L. High-quality three-dimensional holographic display with use of multiple fractional Fourier transform. Chin. Opt. Lett. 2009, 7, 1151-1154. [CrossRef]

21. Zhao, Y.; Cao, L.C.; Zhang, H.; Kong, D.Z.; Jin, G.F. Accurate calculation of computer-generated holograms using angularspectrum layer-oriented method. Opt. Express 2015, 23, 25440-25449. [CrossRef] [PubMed]

22. Arbabi, A.; Horie, Y.; Ball, A.J.; Bagheri, M.; Faraon, A. Subwavelength-thick lenses with high numerical apertures and large efficiency based on high-contrast transmitarrays. Nat. Commun. 2015, 6, 7069. [CrossRef] [PubMed] 